\title{
Spin-orbit interaction and large inelastic rates in bismuth-helium collisions
}

\section{Citation}

Maxwell, S. E., M. T. Hummon, Y. Wang, A. A. Buchachenko, R. V. Krems, and J. M. Doyle. 2008. "Spin-Orbit Interaction and Large Inelastic Rates in Bismuth-Helium Collisions." Physical Review A 78 (4) (October 17). doi:10.1103/physreva.78.042706.

\section{Published Version}

doi:10.1103/PhysRevA.78.042706

\section{Permanent link}

http://nrs.harvard.edu/urn-3:HUL.InstRepos:27868190

\section{Terms of Use}

This article was downloaded from Harvard University's DASH repository, and is made available under the terms and conditions applicable to Other Posted Material, as set forth at http:// nrs.harvard.edu/urn-3:HUL.InstRepos:dash.current.terms-of-use\#LAA

\section{Share Your Story}

The Harvard community has made this article openly available.

Please share how this access benefits you. Submit a story.

\section{Accessibility}




\title{
Spin-orbit interaction and large inelastic rates in bismuth-helium collisions
}

\author{
S. E. Maxwell, ${ }^{1, *}$ M. T. Hummon, ${ }^{1}$ Y. Wang, ${ }^{1}$ A. A. Buchachenko, ${ }^{2,3}$ R. V. Krems, ${ }^{2}$ and J. M. Doyle ${ }^{1}$ \\ ${ }^{1}$ Physics Department, Harvard University, Cambridge, Massachusetts 02138, USA \\ ${ }^{2}$ Department of Chemistry, University of British Columbia, Vancouver, British Columbia V6T 1Z1, Canada \\ ${ }^{3}$ Department of Chemistry, Moscow State University, Moscow 119991, Russia
}

(Received 14 January 2008; published 17 October 2008)

\begin{abstract}
We present a combined experimental and theoretical study of cold collisions between bismuth and helium atoms in strong magnetic fields and demonstrate that the spin-orbit interaction coupling between different nonrelativistic states of Bi leads to rapid Zeeman relaxation. The Zeeman relaxation of Bi in the ground electronic state is found to be very efficient due to the admixture of electronic excited states which show an interaction anisotropy due to their nonzero electronic orbital angular momentum. Our results indicate that dense ensembles of heavy relativistic atoms will generally be unstable in magnetic traps due to significant spin relaxation induced by spin-orbit interactions.
\end{abstract}

DOI: 10.1103/PhysRevA.78.042706

PACS number(s): 34.10.+x, 31.15.aj, 37.10.Gh

\section{INTRODUCTION}

Inelastic collisions between paramagnetic atoms and noble-gas atoms in a magnetic field have long been of significant interest for experiments in optical pumping [1], precision measurement and precision magnetometry $[2,3]$, nuclear physics $[4,5]$, and magnetic resonance imaging [6]. Inelastic collisions of alkali-metal atoms with noble-gas atoms at elevated temperatures were therefore studied in great detail $[5,7-11]$. The interest in collisions of paramagnetic atoms and helium at low temperatures has recently been stimulated by the development of cryogenic cooling techniques, which may allow for enhancement of coherence times in precision measurements $[12,13]$, and the use of cold ( $\$ 1 \mathrm{~K})$ helium buffer gas for loading atoms in a magnetic trap [14-18]. Low-temperature interactions of open-shell atoms with helium are, however, poorly understood. Our recent study of the transition-metal atoms $\mathrm{Ti}$ and $\mathrm{Sc}$ and the atoms from the lanthanide series [14-19] demonstrated that cold-collision experiments using helium buffer gas provide detailed information on the structure of those open-shell atoms and their interaction properties. Here, we present a study of a very different system: cold collisions of helium and bismuth (the heaviest stable element with a half-filled $p$ shell) and discuss how measurements of elastic scattering and collision-induced Zeeman relaxation at low temperatures can provide a probe of the spin-orbit interaction in heavy atoms.

Collisions between paramagnetic atoms and helium can change the spin orientation of the open-shell atom through three different mechanisms: spin exchange, spin-rotation interaction involving the rotational motion of the collision complex, and direct relaxation induced by the anisotropy of the atom-atom interaction [20]. Spin exchange occurs only in collisions with ${ }^{3} \mathrm{He}$ due to the hyperfine interaction between the unpaired valence electrons of the open-shell atom and the spin $I=1 / 2$ of the ${ }^{3} \mathrm{He}$ nucleus. This interaction is weak and our experiments are insensitive to it. The spin-rotation inter-

\footnotetext{
*maxwell@cua.harvard.edu
}

action is induced by the second-order spin-orbit couplings. It can be represented by the phenomenological operator form $\gamma(R) \mathbf{N} \cdot \mathbf{S}$, where $\mathbf{N}$ is the rotational angular momentum of the collision complex and $\gamma(R)$ is the spin-rotation interaction constant $[9,10]$. This interaction is expected to vary quadratically with the temperature $[12,21]$ and thus has little effect in low-temperature collisions.

The anisotropy of the atom-atom electronic interaction, however, can be strong. It arises from the orbital motion of the electrons with nonzero orbital angular momentum [20]. Different orientations of the orbital angular momentum relative to the collision axis give rise to different interaction energies. The effect of electronic interaction anisotropy on cold collisions with helium has been studied for several different atoms $[16-19,22,23]$. It was shown that angular momentum transfer induced by the electronic anisotropy of open-shell atoms, such as oxygen in the ground state ${ }^{3} P_{2}$, is fast.

The lowest-energy electronic term of $\mathrm{Bi}$ is classified as an $S$ state with a spin multiplicity of 4 . The interaction between $S$-state atoms is isotropic [20], and inelastic collisions between an $S$-state atom and helium at low temperatures are forbidden. The $L S$-coupling scheme is, however, insufficient for heavy atoms with strong spin-orbit interactions. Calculations have shown that the nominally $S$ ground state of bismuth has only $\sim 57 \% S$ character [24,25]; contributions to the ground state mixed in by spin-orbit (SO) coupling include terms with nonzero angular momentum. These non- $S$ terms introduce electronic interaction anisotropy with helium. Thus, measurements of collision processes mediated by electronic interaction anisotropy can probe the SO interaction in atoms. We observe the effects of this anisotropy in $\mathrm{Bi}$ experimentally by observing angular momentum reorientation in collisions with cold helium gas.

\section{EXPERIMENT}

Our apparatus is described in detail in Refs. $[18,26]$. In brief, our measurements are performed in a cryogenic buffer gas cell maintained at the temperature $\sim 0.5 \mathrm{~K}$ by a dilution refrigerator. The cell is situated in the bore of a split-coil 
superconducting magnet. We operate the magnet in Helmholtz configuration with magnetic fields of $\sim 1.5 \mathrm{~T}$. Atomic bismuth is produced via laser ablation of a solid elemental target. ${ }^{1}$ The ablation laser is a nanosecond-pulsed Nd:YAG frequency doubled to $532 \mathrm{~nm}$. Typical pulse energies used are $10-20 \mathrm{~mJ}$. The hot atoms produced by the laser ablation are cooled after a characteristic number of collisions with a ${ }^{3} \mathrm{He}$ buffer gas to temperatures of $<1 \mathrm{~K}[14,27]$. The atoms are detected and their density is probed using laser absorption spectroscopy. Buffer gas is introduced into the cell through a $0.6-\mathrm{mm}$-diameter by 8 -mm-long channel. It leaks back out of the cell and is pumped away via the same channel over $\sim 15 \mathrm{~min}$. The buffer gas densities are inferred from a combination of a series of measurements with an atom of known elastic cross section (Nd) and the time after buffer gas introduction. Quoted buffer gas densities are accurate to within a factor of $\sim 2$.

We operate the magnet at sufficiently high field that the nuclear spin is uncoupled from the electronic spin and can effectively be ignored in studies of collision dynamics. We measure and simulate the laser absorption spectra in the Helmholtz field across a wide range of frequencies to identify lines corresponding to different $m_{J}$ states. The lowestenergy Zeeman state $m_{J}=-J$ produces a large signal. Atoms in this state are observable for a time after ablation approximately equal to the field-free diffusion time, $\tau=30-80 \mathrm{~ms}$. Atoms in other states are subject to inelastic losses, and their shorter persistence makes their detection difficult. Nonetheless, the measured spectrum of high-field-seeking states along with our simulated spectrum allowed us to locate and measure the lifetimes after ablation of spectral lines due to $m_{J}>-J$.

To find the rate of inelastic collisions for bismuth, we used a spectral line due to a high-field-seeking (HFS) $\left(m_{J}\right.$ $\left.=-J=-3 / 2, m_{I}=-I=-9 / 2\right)$ state and a line due to a lowfield-seeking (LFS) $\left(m_{J}=J=3 / 2, m_{I}=I=9 / 2\right)$ state at a magnetic field of $1.5 \mathrm{~T}$. After a single insertion of buffer gas into the cell, we alternately measured the decay lifetime of each of these two states, leaving several minutes between measurements to prevent heating of the cell due to repeated ablation pulses. We tuned the laser to a fixed frequency as close to the peak of each spectral line as possible and measured the loss rate $\tau^{-1}$ of the exponential tail of the decay for each state in a narrow (factor of 2) range of buffer gas densities.

To extract the inelastic loss rate $\Gamma_{i n}$, we model the data as $\tau^{-1}=\Gamma_{i n} n_{\mathrm{He}}+\tau_{0}$, where $\tau_{0}$ accounts for all processes leading to loss of the ground state. The magnitude of $\tau_{0}$ should be equal to the diffusion lifetime, but we observe additional loss processes which we attribute to interactions with unidentified particles produced by the ablation pulse. We assume that this loss mechanism does not depend on the state $m_{J}$. For all the data we used to infer $\Gamma_{i n}, \tau_{0} \gg \tau$, so we can approximate $\tau^{-1}=\Gamma_{i n} n_{\mathrm{He}}$. However, a fit to the data of this form indicates that the model is unphysical; the inelastic rate decreases with increasing buffer gas density. Figure 1 shows the dependence of the apparent inelastic rate coefficient on the buffer gas

\footnotetext{
${ }^{1}$ Alfa Aesar stock number 41636. $99.999 \%$ pure. $0.03 \mathrm{ppm} \mathrm{Cu}$, 0.04 ppm K, 0.04 ppm Na.
}

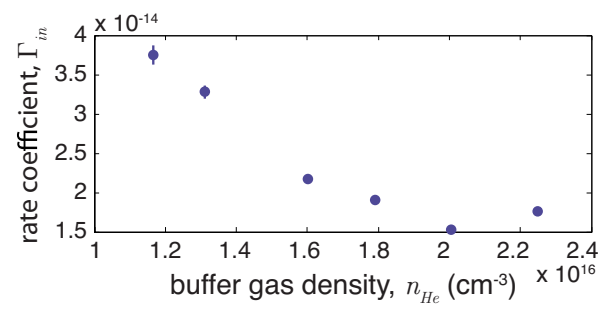

FIG. 1. (Color online) The apparent inelastic rate coefficient $\Gamma_{i n}$ at different buffer gas densities. If we were observing the true inelastic collision rate, rather than cooling of the buffer gas with a Zeeman temperature thermalized with the translational motion at all times, we would expect this plot to be a flat line. This leads us to interpret our data as a lower bound on the inelastic rate coefficient.

density. If this were a measurement of the actual Zeeman relaxation rate, the plot would show a horizontal line. Instead, we observe a curve which decreases rapidly with buffer gas density (as $n_{\mathrm{He}}^{-1.5 \pm 0.2}$ ), consistent with cooling of the buffer gas in a few milliseconds after the ablation. We compared the apparent Zeeman temperature with previous measurements taken in our laboratory [17] and conclude that we are observing cooling of the buffer gas, with the translational and Zeeman temperatures near equilibrium during the measurement. Our measurement can therefore be interpreted as a lower bound of the Zeeman relaxation rate. From the value of the apparent inelastic rate coefficient observed at the lowest buffer gas density, $\quad \Gamma_{\text {in,apparent }}=3.8 \pm .25$ $\times 10^{-14} \mathrm{~cm}^{3} \mathrm{~s}^{-1}$, where the quoted error is a $95 \%$ statistical error bound, and taking into account the systematic uncertainty of $\sim 2$ in the buffer gas density, we conclude that the Zeeman relaxation rate must be $\Gamma_{\text {in }}>1.8 \times 10^{-14} \mathrm{~cm}^{3} \mathrm{~s}^{-1}$. Using the theoretical value of the rate coefficient for elastic collisions, $7 \times 10^{-10} \mathrm{~cm}^{3} \mathrm{~s}^{-1}$, computed as described below, we obtain the elastic-to-inelastic collision ratio of $\gamma<4$ $\times 10^{4}$. For comparison, the elastic-to-inelastic collision ratio for titanium (which has a ${ }^{3} F_{2}$ electronic configuration) in helium was previously measured $[17,18]$ to be $1.3 \pm 0.5$ $\times 10^{4}$. While the value for titanium is considered high for a non- $S$-state atom, the limit measured for bismuth (which is far higher than the theoretical value we describe below) is low for a nominally $S$-state atom. Figure 2 shows the decay of HFS and LFS states of both bismuth and titanium at a buffer gas density of $n_{\mathrm{He}}=1.6 \times 10^{16} \mathrm{~cm}^{-3}$.

\section{THEORY}

In order to verify the validity of our measurements and understand the mechanism of spin relaxation in bismuthhelium collisions, we have performed a detailed analysis of Bi-He scattering based on calculations of the interaction potentials and the SO interaction. The total Hamiltonian of the $\mathrm{Bi}-\mathrm{He}$ collision system in a magnetic field can be written in the form

$$
\hat{H}=-\frac{\hbar^{2}}{2 \mu R} \frac{\partial^{2}}{\partial R^{2}} R+\frac{l^{2}}{2 \mu R^{2}}+\hat{V}_{\mathrm{nr}}+\hat{V}_{\mathrm{SO}}+\hat{V}_{\mathrm{B}},
$$

where $\mu$ is the reduced mass of the collision system, $R$ is the interatomic distance, $\hat{V}_{\mathrm{nr}}$ is the operator describing the non- 


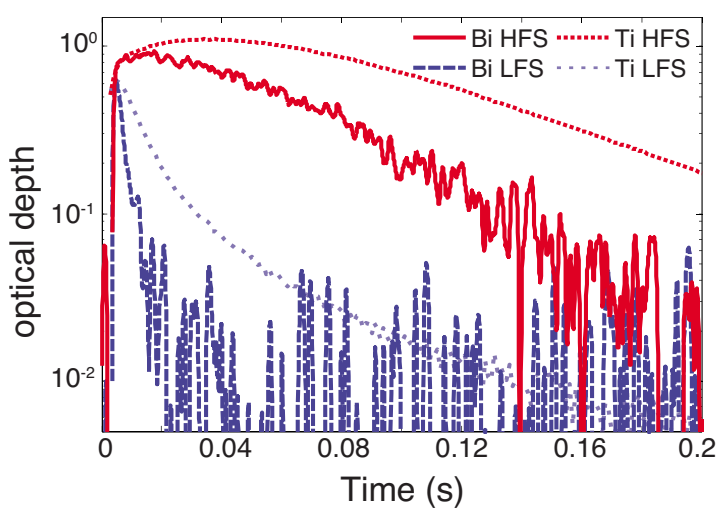

FIG. 2. (Color online) A comparison of the decay of HFS and LFS states of both bismuth and titanium. The traces have been scaled to have the same amplitude at early times. See text for more description of the comparison of these two species.

relativistic electrostatic interaction between the atoms, $l$ is the rotational angular momentum of the nuclei, $\hat{V}_{\text {SO }}$ is the operator of the $\mathrm{SO}$ interaction, and $\hat{V}_{\mathrm{B}}$ describes the interaction of $\mathrm{Bi}$ with external magnetic fields. The valence electron configuration $6 s^{2} 6 p^{3}$ of $\mathrm{Bi}$ in the ground electronic state gives rise to the electronic terms ${ }^{4} S^{\circ},{ }^{2} P^{\circ}$, and ${ }^{2} D^{\circ}$ split by the SO interaction into the following states: ${ }^{4} S_{3 / 2}^{\circ},{ }^{2} D_{3 / 2}^{\circ},{ }^{2} D_{5 / 2}^{\circ}$, ${ }^{2} P_{1 / 2}^{\circ}$, and ${ }^{2} P_{3 / 2}^{\circ}$ [28]. Therefore, we represent the eigenfunctions of the Hamiltonian (1) as follows

$$
\psi=\frac{1}{R} \sum_{L} \sum_{j m_{j}} \sum_{l m_{l}} F_{L j m_{j} l m_{l}}\left|j m_{j}(L)\right\rangle\left|l m_{l}\right\rangle,
$$

where the total orbital angular momentum of the electrons, $L$, can take the value 0,1, or $2, j$ is the vector sum of $L$ and the total electron spin $S$ for a given nonrelativistic state of $\mathrm{Bi}$, and $m_{j}$ and $m_{l}$ are the projections of $j$ and $l$ on the magnetic field axis. The SO interaction couples states with different $L$ and the interaction with magnetic fields $\hat{V}_{B}$ couples states with different $j$, so the scattering problem cannot be solved directly in the basis (2). Following the procedure developed in Ref. [29], we introduce an additional transformation to define a new basis set of functions:

$$
\phi_{\alpha}^{m_{j} l m_{l}}=\sum_{L} \sum_{j} C_{\alpha L j}^{m_{i} l m_{l}}\left|j m_{j}(L)\right\rangle\left|l m_{l}\right\rangle .
$$

The basis transformation $\mathbf{C}$ is constructed numerically from the eigenvectors of the matrix of $\hat{V}_{\mathrm{SO}}+\hat{V}_{\mathrm{B}}$ in the basis (2). The substitution of expansions (2) and (3) into the Schrödinger equation with the Hamiltonian (1) leads to the following system of coupled differential equations:

$$
\begin{aligned}
& {\left[\frac{d^{2}}{d R^{2}}-\frac{l(l+1)}{R^{2}}+2 \mu E\right] F_{\alpha l m_{l}}} \\
& \quad=2 \mu \sum_{\alpha^{\prime} l^{\prime} m_{l}^{\prime}}\left[\mathbf{C}^{T} \mathbf{U} C\right]_{\alpha l m_{l} ; \alpha l^{\prime} m_{l}^{\prime}} F_{\alpha^{\prime} l^{\prime} m_{l}^{\prime}}(R),
\end{aligned}
$$

where $E$ is the total energy of the collision complex and $\mathbf{U}$ represents the sum of the matrices of $\hat{V}_{\mathrm{nr}}, \hat{V}_{\mathrm{SO}}$, and $\hat{V}_{\mathrm{B}}$ in the basis (2).
The matrix of the operator

$$
\hat{V}_{\mathrm{B}}=\mu_{B} B\left(\hat{L}_{z}+2 \hat{S}_{z}\right)
$$

can be evaluated in the basis of direct products $\left|L M_{L}\right\rangle\left|S M_{S}\right\rangle$ related to the basis of coupled functions $\left|j m_{j}(L)\right\rangle$ by the Clebsch-Gordan transformation. Here, $B$ is the magnitude of an external magnetic field, $\mu_{B}$ is the Bohr magneton, $\hat{L}_{z}$ and $\hat{S}_{z}$ are the operators giving the $z$ component of $\hat{L}$ and $\hat{S}$, and $M_{L}$ and $M_{S}$ denote the projections of $\hat{L}$ and $\hat{S}$ on the magnetic field axis.

In order to evaluate the matrices of $\hat{V}_{\mathrm{nr}}$ and $\hat{V}_{\mathrm{SO}}$ in the basis (2), we computed the interaction potentials of the $\mathrm{Bi}-\mathrm{He}$ dimer and the matrix of the SO interaction in the $L S$ basis spanning the electronic states ${ }^{4} S^{\circ},{ }^{2} P^{\circ}$, and ${ }^{2} D^{\circ}$. The interaction of $\mathrm{Bi}$ in these electronic states with $\mathrm{He}$ leads to six adiabatic potentials classified within the $L S$-coupling scheme as ${ }^{4} \Sigma^{-}\left({ }^{4} S^{\circ}\right),{ }^{2} \Delta\left({ }^{2} D^{\circ}\right),{ }^{2} \Pi\left({ }^{2} D^{\circ}\right),{ }^{2} \Sigma^{-}\left({ }^{2} D^{\circ}\right),{ }^{2} \Pi\left({ }^{2} P^{\circ}\right)$, and ${ }^{2} \Sigma^{+}\left({ }^{2} P^{\circ}\right)$. Our calculations were carried out with the MOLPRO package of programs [30]. Sixty inner-shell electrons of $\mathrm{Bi}$ were approximated by the relativistic effective core potential [31], while the remaining electrons were described using an augmented correlation-consistent aug-ccpV5Z basis set [32]. The same basis was used for He [33]. The energies and the wave functions were calculated by the internally contracted multireference configuration interaction (MRCI) method [34]. The reference orbitals were obtained by means of the complete active space self-consistent field (CASSCF) method in which seven electrons were distributed over nine active orbitals representing $6 s 6 p 7 s 7 p$ shells of $\mathrm{Bi}$ and $1 s$ shell of He. CASSCF calculations involved averaging over all nine spatial components of the molecular states. In the MRCI calculations, the orbitals corresponding to the $5 s^{2} 5 p^{6} \mathrm{Bi}$ shells were kept in the core. The total energies were corrected for the basis set superposition error (BSSE) using the supermolecular approach [35] with the same CASSCF+MRCI calculations for the separated $\mathrm{Bi}$ and $\mathrm{He}$ atoms in the full $(\mathrm{Bi}+\mathrm{He})$ basis set. The original MRCI wave functions and the BSSE-corrected interaction energies were used to evaluate the matrix of the SO operator in the spherical components basis of the MOLPRO program. This matrix was then transformed to the $\left|L M_{L}\right\rangle\left|S M_{S}\right\rangle$ basis. To ensure an accurate description of the long-range dispersion interaction, we recomputed the interaction potential for the ground state ${ }^{4} \Sigma^{-}$of the Bi-He complex using the coupled-cluster method with single, double, and noniterative triple excitations $\operatorname{CCSD}(\mathrm{T})[36,37]$, implemented with the same basis set and orbital partitioning and with the restricted Hartree-Fock (RHF) reference wave functions. The results were corrected for the basis-set superposition error. The interaction potentials for the excited states of the Bi-He complex were then obtained by adding the excitation energies from the MRCI calculation.

The validity of this $a b$ initio approach can be verified by examining the asymptotic limit $R \rightarrow \infty$, where the matrix of the SO interaction can be parametrized by six coefficients presented in Table I. $E_{D}$ and $E_{P}$ correspond to the energies of the nonrelativistic states ${ }^{2} D^{\circ}$ and ${ }^{2} P^{\circ} . A_{P}$ and $A_{D}$ represent the SO interaction within the ${ }^{2} P^{\circ}$ and ${ }^{2} D^{\circ}$ states, and $B_{S P}$ and 
TABLE I. $L S$ parameters for $\mathrm{Bi}$ atom $\left(\mathrm{cm}^{-1}\right)$.

\begin{tabular}{lcccccc}
\hline \hline Parameter & $E_{D}$ & $E_{P}$ & $B_{S P}$ & $B_{P D}$ & $A_{D}$ & $A_{P}$ \\
\hline This work & 8944 & 15769 & -8610 & -9637 & -92 & -131 \\
Ref. [24] & 8901 & 14835 & -10093 & -11284 & -101 & -164 \\
\hline \hline
\end{tabular}

$B_{P D}$ represent the coupling strength between the states with different electronic orbital angular momenta. Table I shows that the results of our calculation are in good agreement with the parameters derived from the measurements of atomic energy levels [24]. The calculations reproduced the measured energy levels of atomic $\mathrm{Bi}$ to within $10 \%$ with the largest deviation observed for the highest ${ }^{2} P_{3 / 2}^{\circ}$ level (29 833 vs $33165 \mathrm{~cm}^{-1}$ ) [28]. To verify the accuracy of our calculation of the interaction potentials at long range important for collision dynamics at low temperatures, we computed the static dipole polarizability of $\mathrm{Bi}$ using a finite-field $\operatorname{CCSD}(\mathrm{T})$ method. Our result of 48.8 a.u. is in excellent agreement with the theoretical values recommended in Ref. [38] (49.9 a.u.) and obtained in Ref. [39].

The interaction potentials of the Bi-He complex parametrize the matrix of the $\hat{V}_{\mathrm{nr}}$ interaction. To verify our computer programs, we constructed this matrix using two different methods. The first approach is based on the direct transformation of the total wave function from the moleculefixed to space-fixed coordinate frame. This transformation involves summation over many quantum numbers and is quite cumbersome. An alternative approach is based on the spherical tensor expansion introduced in Ref. [20]. The nonrelativistic interaction potential between $\mathrm{Bi}$ in a particular $L$ state and He can be written in the following form:

$$
\hat{V}_{\mathrm{nr}}^{L}=\sum_{\lambda} V_{\lambda}^{L} \frac{4 \pi}{2 \lambda+1} \sum_{m_{\lambda}} Y_{\lambda m_{\lambda}}^{*}(\hat{R}) Y_{\lambda m_{\lambda}}(\hat{\rho}),
$$

where $\hat{R}$ is the unit vector specifying the direction of the interatomic axis $R, \hat{\rho}$ is the unit vectors describing the rotation of the open-shell electron orbitals, $Y_{\lambda m_{\lambda}}$ are the spherical harmonics, $m_{\lambda}$ is the projection of $\lambda$ on the magnetic field axis, and $V_{\lambda}$ are the expansion coefficients related to the nonrelativistic interaction potentials of the Bi-He complex. For the ground electronic state $L=0$ of $\mathrm{Bi}$, there is only one expansion coefficient $V_{\lambda=0}=V_{4} \Sigma^{-}$. The interaction of He with $\mathrm{Bi}$ in the state with $L=1$ gives rise to two interaction potentials and two expansion coefficients $V_{\lambda}$ related as follows:

$$
\begin{aligned}
& V_{\lambda=0}=\left(2 V^{2} \Pi+V^{2} \Sigma^{+}\right) / 3, \\
& V_{\lambda=2}=5\left(V^{2} \Sigma^{+}-V^{2} \Pi\right) / 3 .
\end{aligned}
$$

Finally, the interaction of $\mathrm{He}$ with $\mathrm{Bi}$ in the state with $L=2$ gives rise to three interaction potentials and three expansion coefficients $V_{\lambda}$ related as follows:

$$
\begin{gathered}
V_{\lambda=0}=\left(V_{2^{-}}+2 V_{2_{\Pi}}+2 V_{{ }}\right) / 5, \\
V_{\lambda=2}=\left(V^{2} \Sigma^{-}-V_{2} \Delta\right)+\left(V^{2} \Pi-V_{2}\right),
\end{gathered}
$$

TABLE II. Equilibrium distances $R_{e}(\AA)$, binding energies $D_{e}$ $\left(\mathrm{cm}^{-1}\right)$, and $C_{6}$ coefficients (a.u.) of the Bi-He nonrelativistic potentials. For the ground ${ }^{4} \Sigma^{-}$potential both $\operatorname{CCSD}(\mathrm{T})$ and MRCI (in parentheses) results are presented; for others, MRCI excitation energies referred to the ground $\operatorname{CCSD}(\mathrm{T})$ potential.

\begin{tabular}{lccc}
\hline \hline State & $R_{e}$ & $D_{e}$ & $C_{6}$ \\
\hline${ }^{4} \Sigma^{-}\left({ }^{4} S^{\circ}\right)$ & $(4.51)$ & $(9.1)$ & $(24.7)$ \\
& 4.64 & 10.0 & 27.0 \\
${ }^{2} \Delta\left({ }^{2} D^{\circ}\right)$ & 4.96 & 6.9 & 29.0 \\
${ }^{2} \Pi\left({ }^{2} D^{\circ}\right)$ & 4.64 & 9.4 & 28.7 \\
${ }^{2} \Sigma^{-}\left({ }^{2} D^{\circ}\right)$ & 4.56 & 9.6 & 28.6 \\
${ }^{2} \Pi\left({ }^{2} P^{\circ}\right)$ & 4.96 & 6.9 & 30.0 \\
${ }^{2} \Sigma^{+}\left({ }^{2} P^{\circ}\right)$ & 4.44 & 11.5 & 29.2 \\
\hline \hline
\end{tabular}

$$
V_{\lambda=4}=9\left(V_{2^{-}}-V_{2}\right) / 5+3\left(V_{2_{\Delta}}-V_{2_{\Pi}}\right) / 5 .
$$

Given these expressions and Eq. (6), the matrix elements of the operator $\hat{V}_{\mathrm{nr}}$ can be readily evaluated in the basis of direct products $\left|L M_{L}\right\rangle\left|l m_{l}\right\rangle$. The terms with $\lambda>0$ represent the electronic interaction anisotropy leading to inelastic collision processes [20]. As follows from Eqs. (7) and (8), the electronic interaction anisotropy is determined by the energy separation of the adiabatic interaction potentials of the collision complex.

Table II presents the equilibrium parameters and longrange $C_{6}$ coefficients for the potentials referred to the ground $\operatorname{CCSD}(\mathrm{T})$ potential energy curve. The nonrelativistic interaction potentials are all very similar and shallow. This indicates that the atoms are bound by weak dispersion forces. The interaction anisotropy in the excited $L \neq 0$ states is also weak. The radial dependence of the parameters describing the SO interaction matrix is depicted in Fig. 3. Note that the interaction with $\mathrm{He}$ induces the SO coupling between the ${ }^{4} \Sigma^{-}\left({ }^{4} S^{\circ}\right)$ and ${ }^{2} \Pi\left({ }^{2} D^{\circ}\right)$ states labeled by $B_{S D}$. This coupling vanishes as the atoms separate. The SO interaction parameters are significantly modified by the interaction with $\mathrm{He}$ only at short distances, well below the equilibrium distance of the interaction potentials. The inset shows, however, that

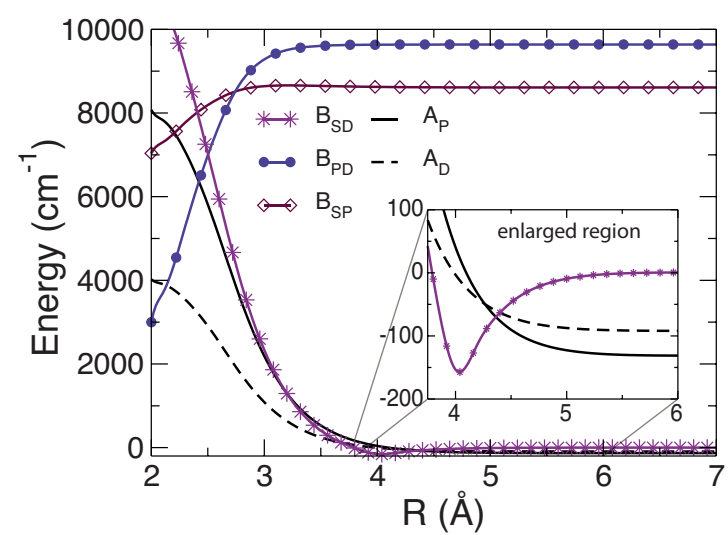

FIG. 3. (Color online) Radial dependence of the SO coupling matrix elements. The inner turning point at $1 \mathrm{~K}$ occurs at $\sim 4.1 \AA$. 


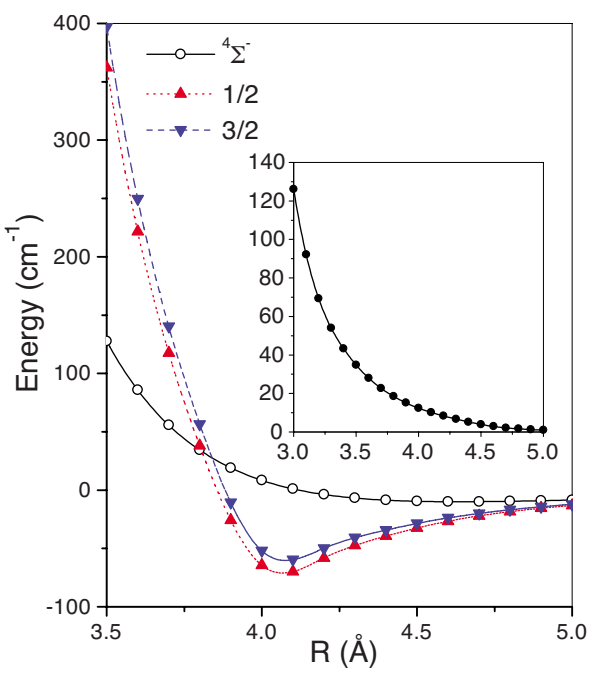

FIG. 4. (Color online) Interaction potentials of the lowest nonrelativistic and SO-coupled states. The inset shows the difference between the $\Omega=1 / 2$ and $3 / 2$ potentials.

at $R=4.1 \AA$, the value roughly equal to the inner turning point of the ${ }^{4} \Sigma^{-}\left({ }^{4} S^{\circ}\right)$ potential for collisions at $1 \mathrm{~K}$ temperature, the variations of the SO interaction parameters are still larger than the binding energies of the interaction potentials.

The effect of the SO interaction on the interaction potentials is illustrated in Fig. 4. The figure presents the potential for the nonrelativistic ${ }^{4} \Sigma^{-}\left({ }^{4} S^{\circ}\right)$ state in comparison with two lowest-energy SO-coupled adiabatic potentials obtained by the diagonalization of the total Hamiltonian matrix. These two potentials correspond to the states with different projections of the total electronic angular momentum on the molecular axis, $\Omega=1 / 2$ and $3 / 2$, correlating with the ground state ${ }^{4} S_{3 / 2}^{\circ}$ of atomic $\mathrm{Bi}$. The $\mathrm{SO}$ coupling enhances the strength of the Bi-He interaction and splits the interaction potential of the ground state. This splitting can be considered as the manifestation of the interaction anisotropy in the total electronic angular momentum. At $R=4.1 \AA$, the splitting is about $10 \mathrm{~cm}^{-1}$.

The numerical integration of Eq. (4) yields the scattering $S$ matrix describing the cross sections for elastic and inelastic scattering [29]. The rate coefficients for elastic and inelastic collisions are calculated from the energy dependence of the cross sections by integration with the Maxwell-Boltzmann distribution function. Figure 5 compares the rate coefficients for elastic collisions with the rates of inelastic scattering summed over all possible relaxation channels. We calculate the elastic-to-inelastic ratio at sub-kelvin collision energies to be about 100, well below the upper limit inferred from the experimental data.

The rate coefficients for Bi-He collisions in the temperature interval between 0.3 and $1.1 \mathrm{~K}$ are affected by a shape resonance whose position is sensitive to small variations of the interaction potentials. For example, a calculation neglecting the radial dependence of the spin-orbit couplings reduces the rate coefficients at $T=0.5 \mathrm{~K}$ by a factor of 3.8 for elastic scattering and by a factor of 1.8 for Zeeman relaxation. To understand the effects of the other SO parameters (see Fig. 3 ), we have repeated calculations of the collision rates with

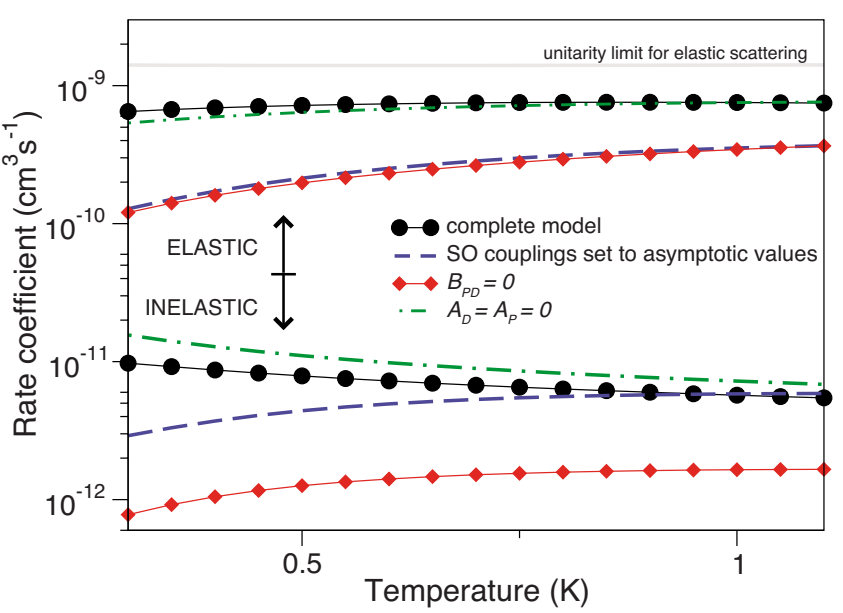

FIG. 5. (Color online) The computed values of the rate coefficients for elastic scattering and Zeeman relaxation in collisions of $\mathrm{Bi}$ with $\mathrm{He}$ at a magnetic field of $1.5 \mathrm{~T}$.

different couplings set to zero. Removing the couplings $A_{D}$ and $A_{P}$ changes the rate constants at $0.5 \mathrm{~K}$ by less than $30 \%$. In contrast, setting $B_{P D}=0$ reduces the inelastic rate by a factor of 6.2 .

\section{SUMMARY}

We have presented the results of measurements and accurate quantum calculations demonstrating that the spin relaxation in cold collisions of $\mathrm{Bi}$ atoms with helium is fast. Inelastic collisions are induced by the spin-orbit interaction in the collision system. Our calculations show that the interaction with $\mathrm{He}$ does not appreciably change the significant spin-orbit interaction parameters at interatomic separations accessible in collisions at sub-kelvin temperatures. An accurate measurement of the Zeeman relaxation of $\mathrm{Bi}$ in cold collisions with He may thus provide a direct probe of the effect on atomic structure of SO interaction mixing nonrelativistic states. Our results, providing a lower bound on the Zeeman relaxation rate in $\mathrm{Bi}-\mathrm{He}$ collisions, indicate that dense ensembles of heavy relativistic atoms will generally be unstable in magnetic traps due to significant spin relaxation induced by SO interactions.

Inelastic collisions between $\mathrm{Bi}$ and $\mathrm{He}$ are induced by an admixture of ${ }^{2} P^{\circ}$ and ${ }^{2} D^{\circ}$ atomic states in the ground state of the collision complex. This admixture is determined by the matrix elements $B_{S P}, B_{P D}$, and $B_{S D}$ of the SO interaction. To lowest nonzero order, the interaction anisotropy between ground-state $\mathrm{Bi}$ and $\mathrm{He}$ is given by $\Delta(R)=\frac{2}{3} \frac{B_{S P}^{2}}{E_{P}^{2}}\left[V_{P \Pi}(R)\right.$ $\left.-V_{P \Sigma}(R)\right]$, where $V_{P \Pi}$ and $V_{P \Sigma}$ are the interaction potentials between $\mathrm{Bi}\left({ }^{2} P^{\circ}\right)$ and He. Thus, the SO interaction transfers the anisotropy of the $P$ state (and $D$ state at higher orders) to the ground $S$ state. We have verified by a test calculation that spin relaxation cannot occur if the parameters $B_{S P}$ and $B_{S D}$ are both set to zero.

We also note that we attempted to trap atomic rhenium $(Z=75)$, which has a ground state with a half-filled $d$ orbital (electronic configuration is $[\mathrm{Xe}] 4 f^{14} 5 d^{5} 6 s^{2}$ ) [40]. The ground state is nominally ${ }^{6} S_{5 / 2}$ and has a Landé $g$ factor of 1.95 [40]. 
We expected that the outer $6 s$ electrons would shield anisotropy due to spin-orbit interactions in the $d$ electrons, thus suppressing inelastic collisions. However, we found a weak upper bound on the elastic to inelastic collision ratio of $\gamma$ $<3 \times 10^{5}$, which proved too high for buffer gas loading of our magnetic trap. Improved detection and loading of atomic rhenium may lead to an improved measurement of $\gamma$.

\section{ACKNOWLEDGMENTS}

We are grateful to Eite Tiesinga for helpful input and we acknowledge support from the Natural Science and Engineering Research Council of Canada, the Russian Basic Research Fund under Project No. 08-03-00414, and the National Science Foundation under Grant No. PHY0457047.
[1] W. Happer, Rev. Mod. Phys. 44, 169 (1972).

[2] D. Budker et al., Rev. Mod. Phys. 74, 1153 (2002).

[3] D. Kimball et al., in Art and Symmetry in Experimental Physics, edited by Dmitry Budker ,Stuart J. Freedman , and Philip H. Bucksbaum, AIP Conf. Proc. No. 596 (AIP, Melville, NY, 2001), pp. 108-122.

[4] W. Happer and W. Van Wijngaarden, Hyperfine Interact. 38, 435 (1987).

[5] M. E. Wagshul and T. E. Chupp, Phys. Rev. A 49, 3854 (1994).

[6] H. Middleton et al., Magn. Reson. Med. 33, 271 (1995).

[7] N. Beverini, P. Minguzzi, and F. Strumia, Phys. Rev. A 4, 550 (1971).

[8] F. A. Franz and C. Volk, Phys. Rev. A 26, 85 (1982).

[9] R. M. Herman, Phys. Rev. 136, A1576 (1964).

[10] T. G. Walker, J. H. Thywissen, and W. Happer, Phys. Rev. A 56, 2090 (1997).

[11] A. B. Baranga, S. Appelt, M. V. Romalis, C. J. Erickson, A. R. Young, G. D. Cates, and W. Happer, Phys. Rev. Lett. 80, 2801 (1998).

[12] A. Sushkov, Ph.D. thesis, University of California, Berkeley, 2006.

[13] A. Hatakeyama, K. Oe, K. Ota, S. Hara, J. Arai, T. Yabuzaki, and A. R. Young, Phys. Rev. Lett. 84, 1407 (2000).

[14] J. Kim, B. Friedrich, D. P. Katz, D. Patterson, J. D. Weinstein, R. DeCarvalho, and J. M. Doyle, Phys. Rev. Lett. 78, 3665 (1997).

[15] J. D. Weinstein, R. deCarvalho, J. Kim, D. Patterson, B. Friedrich, and J. M. Doyle, Phys. Rev. A 57, R3173 (1998).

[16] C. Hancox et al., Nature (London) 431, 281 (2004).

[17] C. I. Hancox, S. C. Doret, M. T. Hummon, R. V. Krems, and J. M. Doyle, Phys. Rev. Lett. 94, 013201 (2005).

[18] C. I. Hancox, Ph.D. thesis, Harvard University, 2005.

[19] R. V. Krems, J. Klos, M. F. Rode, M. M. Szczesniak, G. Chalasinski, and A. Dalgarno, Phys. Rev. Lett. 94, 013202 (2005).

[20] R. V. Krems, G. C. Groenenboom, and A. Dalgarno, J. Phys.
Chem. A 108, 894 (2004).

[21] R. V. Krems, A. Dalgarno, N. Balakrishnan, and G. C. Groenenboom, Phys. Rev. A 67, 060703(R) (2003).

[22] R. V. Krems and A. A. Buchachenko, J. Chem. Phys. 123, 101101 (2005).

[23] R. V. Krems and A. Dalgarno, Phys. Rev. A 68, 013406 (2003).

[24] D. A. Landman and A. Lurio, Phys. Rev. A 1, 1330 (1970).

[25] J. Dembczynski et al., Z. Phys. A 310, 27 (1983).

[26] J. Weinstein, Ph.D. thesis, Harvard University, 2002.

[27] R. deCarvalho et al., Eur. Phys. J. D 7, 289 (1999).

[28] S. George, J. H. Munsee, and J. Vergès, J. Opt. Soc. Am. B 2, 1258 (1985).

[29] R. V. Krems and A. Dalgarno, J. Chem. Phys. 120, 2296 (2004).

[30] H. J. Werner et al., Computer programme MOLPRO, versions 2002.6 and 2006.1, a package of ab initio programs 2006, URL http://www.molpro.net.

[31] W. Küchle et al., Mol. Phys. 74, 1245 (1991).

[32] K. A. Peterson, J. Chem. Phys. 119, 11099 (2003).

[33] D. E. Woon and T. H. Dunning, Jr., J. Chem. Phys. 100, 2975 (1994).

[34] H.-J. Werner and P. J. Knowles, J. Chem. Phys. 89, 5803 (1988).

[35] S. F. Boys and F. Bernardi, Mol. Phys. 19, 553 (1970).

[36] J. D. Watts, J. Gauss, and R. J. Bartlett, J. Chem. Phys. 98, 8718 (1993).

[37] P. J. Knowles, C. Hampel, and H. J. Werner, J. Chem. Phys. 99, 5219 (1993). P. J. Knowles, C. Hampel, and H. J. Werner, ibid. 112, 3106 (2000).

[38] T. M. Miller, in CRC Handbook of Chemistry and Physcis, 83rd ed., edited by D. R. Lide (CRC Press, Boca Raton, FL, 2002), pp. 10-160.

[39] B. O. Roos, R. Lindh, P.-Å. Malmqvist, V. Veryazov, and P. O. Widmark, J. Phys. Chem. A 108, 2851 (2004).

[40] R. E. Trees, Phys. Rev. 112, 165 (1958). 